\section{Mehr Sicherheit durch die Therapieallergene-Verordnung}

Seit dem November 2008 gilt in Deutschland die TherapieallergeneVerordnung. Diese regelt, dass die häufigsten Allergene zur spezifischen Immuntherapie in Zukunft einer Zulassung bedürfen. Welche praktischen Auswirkungen dies hat, erklärt Prof. Dr. Randolf Brehler.

? Was ist das Anliegen der Therapieallergene-Verordnung?

Brehler: Die Verordnung regelt, dass häufig verwendete Allergenextrakte, wie Süßgräser, Baumpollen, Hausstaubmilben, Bienen- und Wespengift, dem normalen Zulassungsprozedere unterzogen werden müssen. Ziel ist der Nachweis der Sicherheit und Effektivität unter Studienbedingungen. Als Allergologe begrüße ich die Neuregelung, da wir sicher sein können, dass künftig nur Extrakte auf den Markt kommen, deren Wirksamkeit belegt werden konnte.

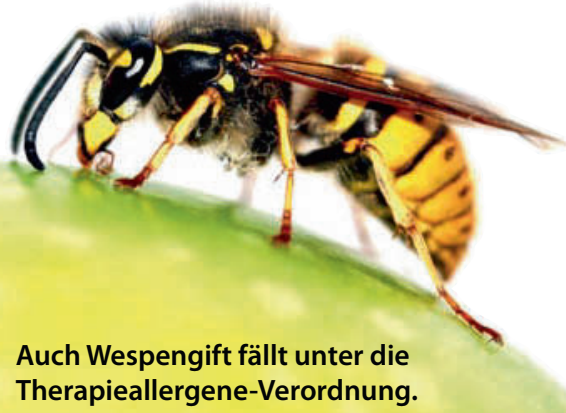

○) Jon Le-Bon / Fotolia.com
? Was hat sich in der bisherigen Praxis geändert?

Brehler: Die Herstellerfirmen mussten bis zum 1. Dezember 2010 für Präparate, die zugelassen werden sollen, die entsprechenden Anträge bei den Behörden vorlegen. Zur Behebung von Mängeln gibt es dann ein weiteres Jahr Zeit. Im Anschluss müssen entsprechende Studien durchgeführt werden. Bereits zugelassene Allergene können weiter so verschrieben werden wie bisher.

? Firmen wie Allergopharma bieten einen großen Teil an bereits zugelassenen Fertigarzneimitteln an. Welchen Vorteil hat dies für die tägliche Praxis?

Brehler: Da die Zulassungen bereits vorliegen, kann von einer langfristigen Verfügbarkeit dieser Produkte ausgegangen werden. Ihre Effektivität und Sicherheit ist entsprechend der früher gültigen Bedingungen geprüft worden. Ebenso ist der vorliegende Zulassungsstatus erfahrungsgemäß bei der Diskussion der Erstattungsfähigkeit eine gute Argumentationshilfe.

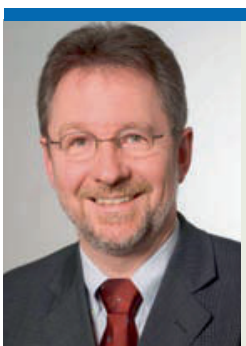

„Persönlich glaube ich, dass viele Präparate vom Markt verschwinden werden."

Prof. Dr. Randolf Brehler Klinik und Poliklinik für Hautkrankheiten, Universitätsklinikum Münster

? Hyposensibilisierungen sind langfristige Behandlungen. Kann der Arzt jetzt verlässlich planen, dass er die Therapie mit einem noch nicht zugelassenen Präparat bis zu Ende durchführen kann?

Brehler: Diese Frage ist momentan nur schwer zu beantworten. Es ist beispielsweise vorstellbar, dass ein Hersteller zwar eine Zulassung anstrebt, diese dann aber aus Kostengründen nicht durchführen kann. Auch kann nicht vorausgesagt werden, ob für alle Präparate der Wirksamkeitsnachweis in den geforderten Studien gelingen wird. Persönlich glaube ich, dass viele Präparate vom Markt verschwinden werden.

! Herr Professor Brehler, vielen Dank für das Gespräch.

Das Interview führte Bettina Reich.

\title{
Psoriasisarthritis: Golimumab hilft nachhaltig
}

— Für die Diagnose und Klassifikation der Psoriasisarthritis (PsA) haben sich in den vergangenen Jahren die CASPAR-Kriterien als klinisch gut anwendbar und valide etabliert. Sie eignen sich nicht nur für Patienten mit länger bestehender Erkrankung, sondern auch für solche mit kurzer Krankheitsanamnese. In einer Untersuchung mit mehr als 100 Patienten mit früher PsA (Beschwerdedauer $<2$ Jahre, DMARD [", disease modifying antirheumatic drugs"]-naiv) lag die Sensitivität der CASPAR-Kriterien bei $87,4 \%$, die Spezifität betrug $99,1 \%$. Diese Zahlen nannte Prof. Dr. Hendrik Schulze-Koops von der Rheumaeinheit der Universität Münster bei einem „Meet the Expert" im Rahmen des TNFa-Forums 2011 in München.

Dass Patienten mit PsA auf die Behandlung mit TNF-Inhibitoren klinisch gut ansprechen, ist aus diversen Untersuchungen bekannt. Zudem belegen Langzeitdaten verschiedener TNF-Blocker die anhaltend gute Wirksamkeit auf den Haut- und Gelenkbefall bei PsA-Patienten.

In der Studie GO-REVEAL führte Golimumab (Simponi ${ }^{\oplus}$ ) $50 \mathrm{mg}$ zu einem anhaltenden Ansprechen nach den Kriterien des American College of Rheumatology (ACR) über zwei Jahre. Auch die langfristige Hemmung der radiologischen Progression ist für Golimumab belegt: Wie Daten vom letztjährigen Kongress des ACR zeigen, nahm in der Gruppe mit Golimumab $50 \mathrm{mg}$ + MTX innerhalb von zwei Jahren der Gesamt-vdHS-Score um 0,4 Punkte ab, während er in der Gruppe mit initialen Gabe von MTX + Placebo um 0,1 Punkte stieg. Nach zweijähriger Golimumab-Therapie zeigte etwa jeder zweite Patient sogar ein PASI90-Ansprechen, rund $60 \%$ der Patienten erzielten im Health Assessment Questionnaire einen Wert $\leq 0,5$.

Dr. Brigitta Schneider

TNF $\alpha$-Forum, Meet the Expert: Remicade ${ }^{\oplus}$ und Simponi ${ }^{\oplus}$ das TNFa-Prinzip im Einsatz. München, 21. Januar 2011 (Veranstalter: Essex/MSD) 